\title{
Variación espacio-temporal de la fecundidad de Keratella cochlearis (Rotifera) en un embalse meso-eutrófico: Importancia relativa del alimento y de la depredación por copépodos.
}

\author{
Eloísa Ramos-Rodríguez ${ }^{1}$ y José M. Conde-Porcuna² \\ ${ }^{1}$ Departamento de Biología Animal y Ecología, Facultad de Ciencias, Universidad de Granada, 18071 Granada. \\ eloisa@ugr.es \\ 2Instituto del Agua, Universidad de Granada, Ramón y Cajal 4, 18071 Granada.jmconde@ugr.es
}

\begin{abstract}
RESUMEN
Se ha estudiado la distribución espacio-temporal de la fecundidad de una población del rotífero cosmopolita, Keratella cochlearis, en el embalse de Iznájar (Córdoba) en relación con la temperatura, la concentración de alimento y la presencia de copépodos depredadores (Neolovenula alluaudi y Acanthocyclops vernalis). Este estudio se llevó a cabo durante la primavera de 1997 mediante un muestreo de alta frecuencia (cada 3 días), tomando muestras en superficie, $4 \mathrm{~m}$ y $10 \mathrm{~m}$ de profundidad. Los resultados obtenidos sugieren que la fecundidad de $K$. cochlearis muestra un patrón de distribución espacio-temporal dependiente de la concentración de alimento y de la presencia de copépodos depredadores adultos. La fecundidad de K. cochlearis en el embalse de Iznájar podría estar regulada por la concentración de alimento en aquellos momentos en que la biomasa de copépodos depredadores es relativamente baja. Sin embargo, en los periodos en que la biomasa de copépodos depredadores es elevada, la fecundidad de Keratella parece estar principalmente controlada por la presencia de dichos copépodos. Estos aspectos deberían ser considerados en aquellos estudios sobre dinámica de poblaciones zooplanctónicas de sistemas acuáticos.
\end{abstract}

Palabras clave: distribución espacio-temporal, fecundidad, Keratella cochlearis, Rotifera, depredación, copépodos.

\section{ABSTRACT}

We have studied the spatio-temporal distribution of the fecundity of a population of the cosmopolitan rotifer, Keratella cochlearis, in Iznájar Reservoir (Córdoba, South of Spain) in relation to temperature, food concentration and the presence of predatory copepods (Neolovenula alluaudi and Acanthocyclops vernalis). This study was carried out during spring of 1997 using an intensive sampling design (every 3 days). We collected samples at surface, $4 \mathrm{~m}$ and $10 \mathrm{~m}$ depth. Results suggest that the fecundity of $\mathrm{K}$. cochlearis shows a spatio-temporal distribution dependent on the food concentration and the presence of adult predatory copepods. Fecundity of $\mathrm{K}$. cochlearis in Iznájar Reservoir could be regulated by food concentration when the biomass of predator copepods is relatively low. However, when biomass of predatory copepods is high, the fecundity of Keratella seems to be mainly regulated by the presence of copepods. These aspects should be considered in studies on the dynamics of zooplankton populations of aquatic systems.

Keywords: spatio-temporal distribution, fecundity, Keratella cochlearis, Rotifera, predation, copepods.

\section{INTRODUCCIÓN}

En ecología, uno de los aspectos de mayor interés desde siempre ha sido, y sigue siendo, el estudio de los posibles factores bióticos y abióticos que regulan la dinámica y estructura de las comunidades naturales. Uno de los principales problemas con los que se enfrenta la ecología es cono- cer las interacciones entre estos factores y cuantificar su importancia relativa, la cual puede variar entre sistemas biológicos e incluso dentro de un mismo sistema (Hunter \& Price, 1992). Entre los factores más importantes que regulan las poblaciones de rotíferos en los sistemas naturales se encuentran el alimento y la temperatura (Edmonson, 1965; Armengol et al., 1998; Ooms- 
Wilms et al., 1999). Sin embargo, numerosos estudios han mostrado también la importancia de la depredación por copépodos y rotíferos carnívoros (Asplanchna) (p. e. Stemberger \& Evans, 1984; Conde-Porcuna \& Declerck, 1998; Dieguez \& Gilbert, 2002), y de la competencia explotativa e interferencia mecánica con grandes dáphnidos ( $>=1.2 \mathrm{~mm}$ ) (p. e. Gilbert, 1988; Conde-Porcuna et al., 1994), sobre el control de las poblaciones de rotíferos en la naturaleza.

Yan et al. (1991) establecieron que la importancia de la depredación por invertebrados sobre el control de las poblaciones de rotíferos es dependiente de la producción de rotíferos. De este modo, los depredadores podrían ser capaces de controlar la abundancia de rotíferos si sus tasas de reproducción estuvieran limitadas por el alimento. En este sentido, Paul \& Schindler (1994) observaron que la baja disponibilidad de alimento en los lagos alpinos oligotróficos podría reducir las tasas reproductivas de los rotíferos lo suficiente como para permitir el control de estas poblaciones por depredadores invertebrados (copépodos del género Hesperodiaptomus). Sin embargo, Conde-Porcuna \& Declerck (1998) sugirieron que las poblaciones de rotíferos podrían estar reguladas por depredadores en sistemas eutróficos, sin limitación alguna de alimento.

El hecho de que las especies de Keratella sean resistentes a la depredación por copépodos ciclópidos y calanoides se ha observado en numerosos estudios: sus lórigas y espinas las protegen contra la depredación (Stemberger \& Evans, 1984; Williamson \& Butler, 1986; Roche, 1990; Plaßmann et al., 1997). Sin embargo, los huevos de $K$. cochlearis no están protegidos por la lóriga $y$, hasta el momento, se ha investigado poco sobre la interacción entre rotíferos grávidos y copépodos depredadores, y sus consecuencias sobre la fecundidad de los rotíferos. Algunos estudios experimentales han indicado que los copépodos y Asplanchna pueden reducir dicha fecundidad consumiendo los huevos de rotíferos y/o consumiendo selectivamente hembras ovígeras (Williamson \& Butler, 1986; Conde-Porcuna \& Sarma, 1995; LeBlanc et al., 1997; Conde-Porcuna \& Declerck, 1998).
En el presente estudio se pretende analizar, en un sistema natural, mediante una intensa campaña de muestreo, la variación espacio-temporal de la fecundidad del rotífero cosmopolita Keratella cochlearis, en relación con su alimento, temperatura y la presencia de crustáceos depredadores y/o competidores.

\section{METODOLOGÍA}

El embalse de Iznájar (2500 ha de área superficial y $980 \cdot 10^{6} \mathrm{~m}^{3}$ de capacidad) está situado en el curso del río Genil, un afluente del río Guadalquivir, en el sur de España. Este embalse, según la concentración media anual de clorofila$a$ total $(20.02 \mu \mathrm{g} / 1) \mathrm{y}$ de fósforo total $(49.63 \mu \mathrm{g} / 1)$, además de los valores medios de transparencia del agua según el disco de Secchi (3.43 m) (Cruz-Pizarro et al., datos no publicados), podría catalogarse como meso-eutrófico (OCDE, 1982).

El periodo de estudio comprendió desde el 5 de abril hasta el 1 de junio de 1997, realizándose muestreos cada tres días entre las 12:00 y 14:00 horas locales. El punto de muestreo (profundidad aprox. $40 \mathrm{~m}$ ) se situó en las proximidades de la presa en un ambiente de características esencialmente pelágicas y una boya señaló su posición durante el estudio. Se recogieron muestras a tres profundidades (superficie, $4 \mathrm{y}$ 10 metros) con una botella Niskin (8 litros), para cuantificar la concentración de clorofila- $a$ correspondiente a la fracción algal $<40 \mu \mathrm{m}$ (fracción algal comestible por el zooplancton), y para caracterizar la comunidad zooplanctónica. También se midió la temperatura del agua in situ en cada una de las profundidades seleccionadas con una sonda "Windermer Profiler". El contenido en clorofila- $a$ se determinó a partir de extractos metanólicos analizados según el método espectrofotométrico propuesto por Talling \& Driver (1961). La biomasa algal ( $\mu \mathrm{g}$ peso seco/ml) se estimó multiplicando el contenido en clorofila- $a(\mu \mathrm{g} / 1)$ por un factor de 0.067 , suponiendo que la clorofila- $a$ constituye, como media, un $1.5 \%$ de el peso seco de la materia orgánica de las algas (APHA, 1992). 
Las muestras de zooplancton (8 1 de agua filtrados por una malla de $40 \mu \mathrm{m}$ ) se fijaron con formol (4\%) azucarado, y posteriormente fueron cuantificadas en un microscopio invertido. Además se tomaron medidas de la longitud del cuerpo de cladóceros y copépodos (10-100 individuos por muestra) y de $K$. cochlearis (30 individuos por muestra) con un micrómetro ocular calibrado. Se consideraron como copépodos adultos aquellos organismos del calanoide Neolovenula alluaudi con longitud $>=1.6 \mathrm{~mm}$, y del ciclópido Acanthocyclops vernalis los individuos con longitud $>=1 \mathrm{~mm}$ (Dussart, 1967). Las biomasas de Daphnia y de los copépodos adultos se estimaron a partir de las densidades y del peso medio individual obtenido a partir de las siguientes fórmulas descritas por Bottrell et al. (1976):

- Daphnia longispina:

- Copépodos:

$$
\mathrm{Ln} \mathrm{W}=1.37+2.5567 \times \mathrm{Ln} \mathrm{L}
$$

$$
\mathrm{Ln} \mathrm{W}=1.9526+2.3990 \times \mathrm{Ln} \mathrm{L}
$$

donde $\mathrm{W}$ es el peso seco medio individual $(\mu \mathrm{g}) \mathrm{y}$ $\mathrm{L}$ es la longitud del cuerpo (mm). El peso seco medio de $K$. cochlearis se estimó a partir del volumen medio individual, obtenido con la fórmula descrita por Pourriot \& Rougier (1991), asumiendo una densidad de 1 (es decir, $1 \mu \mathrm{m}^{3}=$ $\left.10^{-6} \mu \mathrm{g}\right)$ y una relación peso seco/peso fresco de 0.1 (Bottrell et al., 1976).

En cada una de las muestras también se determinó la fecundidad de K. cochlearis como la razón entre el número de huevos y el $\mathrm{n}^{\circ} \mathrm{de}$ individuos.

\section{Análisis estadísticos}

Los análisis estadísticos se llevaron a cabo usando el programa STATISTICA (Statsoft Inc., 1998). Para evaluar el grado de asociación entre la fecundidad de $K$. cochlearis y la concentración de alimento disponible en el sistema se realizaron análisis de correlación de Pearson. Para comparar valores medios de distintas variables entre dos periodos de tiempo se usaron pruebas $t$ de Student, habiéndose comprobado previamen- te la homogeneidad de varianzas y la normalidad de las variables (Sokal \& Rohlf, 1995). Y por último, para comparar valores medios de las variables entre dos profundidades se realizaron pruebas $t$ de Student para muestras pareadas.

\section{RESULTADOS}

Las especies de rotíferos más abundantes en el embalse de Iznájar durante la primavera de 1997 fueron principalmente Keratella cochlearis, Polyarthra vulgaris-dolichoptera y Synchaeta oblonga (Fig. 1a). Polyarthra y Synchaeta sólo alcanzaron densidades importantes durante las
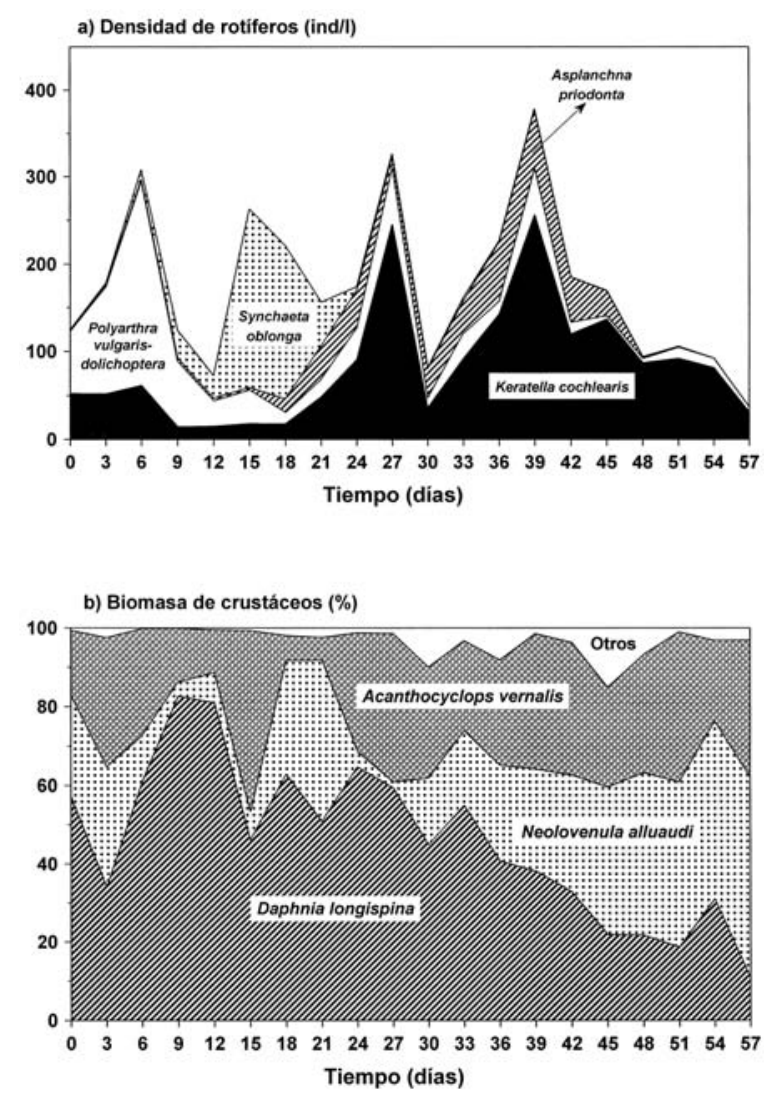

Figura 1. Densidad media de las principales especies de rotíferos (a) y contribución relativa de cladóceros y copépodos a la biomasa total de crustáceos (b), durante la primavera de 1997 en el embalse de Iznájar. Mean density of main rotifer species (a), and relative contribution of cladocerans and copepods to total biomass of crustaceans (b), during spring of 1997 in Iznájar Reservoir. 


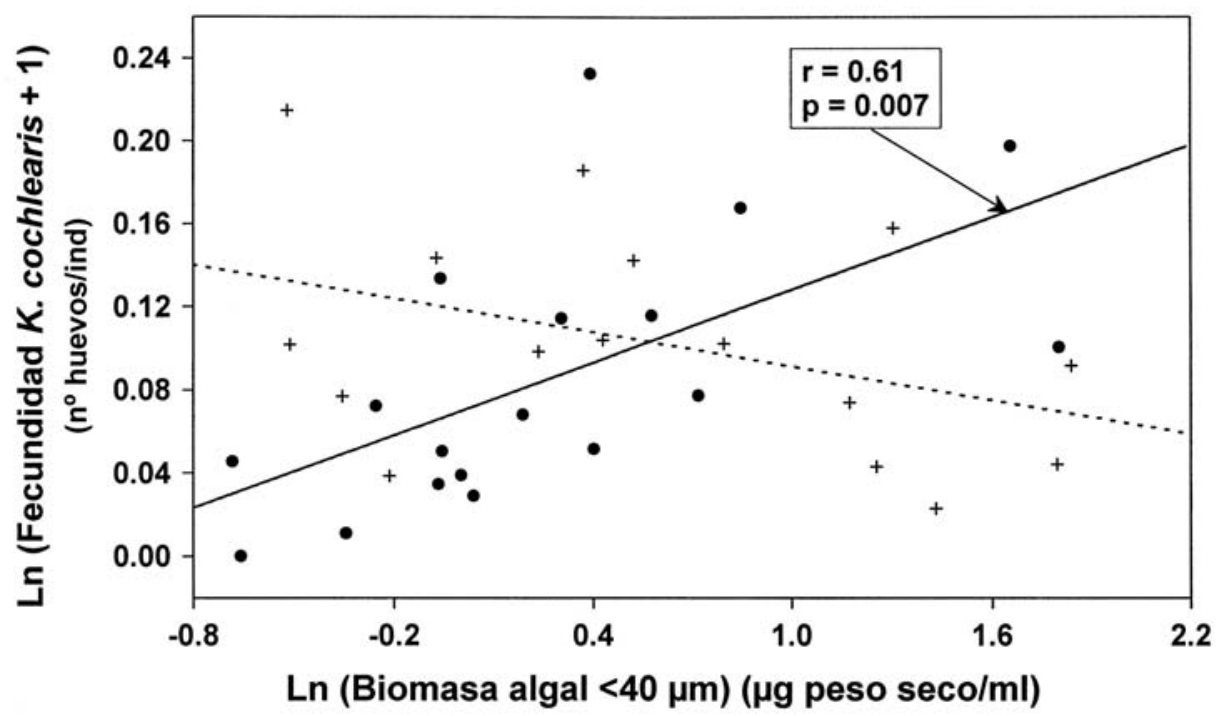

Figura 2. Correlaciones de Pearson entre la fecundidad de K. cochlearis y la biomasa algal $(<40 \mu \mathrm{m})$, para valores de biomasa de copépodos adultos $<5 \mu \mathrm{g} / 1$ (círculos) y $>5 \mu \mathrm{g} / 1$ (cruces). Las correlaciones se restringieron a los casos en los que la densidad de K. cochlearis era superior a $30 \mathrm{ind} / 1$, y la biomasa algal superior a $0.5 \mu \mathrm{g} / \mathrm{ml}$. Pearson correlations between the fecundity of $\mathrm{K}$. cochlearis and the algal biomass $(<40 \mu \mathrm{m})$, for copepod biomass values $<5 \mu \mathrm{g} / \mathrm{l}$ (circles) and $>5 \mu \mathrm{g} / \mathrm{l}$ (crosses). Correlations were restricted to times when $\mathrm{K}$. cochlearis densities were $>30$ ind $/$ and algal biomass was $>0.5 \mu \mathrm{g} / \mathrm{ml}$.

dos primeras semanas del estudio, mientras que Keratella fue la única especie de rotífero que mantuvo densidades relativamente altas durante todo el periodo. Entre los crustáceos, las especies más importantes fueron el cladócero Daphnia longispina y los copépodos Neolovenula alluaudi y Acanthocyclops vernalis. Las otras especies de crustáceos (Bosmina longirostris y Tropocyclops prasinus) nunca tuvieron repercusión destacable en la biomasa (Fig. 1b). La comunidad fitoplanctónica estuvo dominada por algas verdes, criptofíceas y diatomeas (Pérez-Martínez \& SánchezCastillo, datos no publicados).

Durante el periodo de estudio se observó una correlación positiva y significativa entre la fecundidad de $K$. cochlearis y la biomasa algal $(<40 \mu \mathrm{m})$, para aquellos casos en que la biomasa de copépodos adultos de Neolovenula y Acanthocyclops era inferior a $5 \mu \mathrm{g} / 1$ (Fig. 2). Sin embargo, cuando la biomasa de estos copépodos adultos alcanzaba valores superiores a $5 \mu \mathrm{g} / 1$, la relación entre la fecundidad del rotífero y la biomasa algal dejaba de ser significativa (Fig. 2; $\mathrm{r}=-0.40, \mathrm{p}>0.05)$. Las correlaciones se restringieron a los casos en que la densidad de $K$. co- chlearis era superior a 30 ind/1, para evitar posibles errores en la estima de la fecundidad de Keratella por baja densidad de individuos (Conde-Porcuna \& Declerck, 1998). En cualquier caso, se obtuvieron resultados similares cuando se consideraron todas las densidades de $K$. cochlearis para biomasa de copépodos inferior a $5 \mu \mathrm{g} / 1(\mathrm{n}=26 ; \mathrm{r}=0.52 ; \mathrm{p}<0.01)$ y superior a $5 \mu \mathrm{g} / \mathrm{l}(\mathrm{n}=25 ; \mathrm{r}=-0.28 ; \mathrm{p}>0.05)$. Además, todas estas correlaciones se restringieron a aquellos momentos del periodo de estudio en que la biomasa algal comestible por el zooplancton era superior a $0.5 \mu \mathrm{g} / \mathrm{ml}$. Esta concentración es 10 suficientemente elevada como para que la población de $K$. cochlearis no esté limitada por el alimento en este sistema, ya que previamente se ha observado que un clon de $K$. cochlearis, aislado de este embalse, alcanzó tasas de crecimiento de $0.35 \mathrm{ind} / \mathrm{d}$ a concentraciones de alimento comprendidas entre 0.32-0.64 $\mu \mathrm{g} / \mathrm{ml}$ (RamosRodríguez \& Conde-Porcuna, datos no publicados). Estas tasas de crecimiento se encuentran entre los valores máximos descritos para $K$. cochlearis en situaciones naturales (Edmondson, 1965; Zimmerman, 1974). 
Tabla 1. Valores medios $( \pm \mathrm{DE})$ de la fecundidad de $K$. cochlearis $\left(\mathrm{n}^{\circ}\right.$ huevos/ind), concentración de clorofila- $a<40 \mu \mathrm{m}(\mu \mathrm{g} / \mathrm{l})$, biomasa de copépodos adultos $(\mu \mathrm{g} / \mathrm{l})$, biomasa de individuos de Daphnia $\geq 1.2 \mathrm{~mm}(\mu \mathrm{g} / \mathrm{l})$ y temperatura $\left({ }^{\circ} \mathrm{C}\right)$, para cada periodo considerado. Las medias entre periodos fueron comparadas usando una prueba t de Student. Mean values $( \pm S D)$ of the fecundity of K. cochlearis $\left(n^{\circ}\right.$ eggs $/$ ind $)$, chlorophyll-a concentration $<40 \mu \mathrm{m}(\mu \mathrm{g} / \mathrm{l})$, biomass of adult copepods $(\mu \mathrm{g} / \mathrm{l})$, biomass of Daphnia individuals $\geq 1.2 \mathrm{~mm}(\mu \mathrm{g} / \mathrm{l})$ and temperature $\left({ }^{\circ} \mathrm{C}\right)$, for each studied period. Means between periods were compared using a Student's t-test.

\begin{tabular}{lccccc}
\hline & Periodo $\mathbf{1}(\mathbf{n}=\mathbf{1 3})$ & Periodo $\mathbf{2}(\mathbf{n}=\mathbf{7})$ & t & gl & $\mathbf{p}$ \\
\hline Fecundidad de $\boldsymbol{K}$. cochlearis & $0.12 \pm 0.06$ & $0.11 \pm 0.05$ & 0.24 & 18 & $\mathrm{~ns}$ \\
Clorofila- $\boldsymbol{a}<\mathbf{4 0} \boldsymbol{\mu m}$ & $15.45 \pm 7.59$ & $37.08 \pm 12.18$ & -4.93 & 18 & $<0.001$ \\
Biomasa copépodos adultos & $6.29 \pm 8.95$ & $16.04 \pm 8.21$ & -2.39 & 18 & $<0.05$ \\
Biomasa Daphnia $>\mathbf{1 . 2} \mathbf{~ m m}$ & $20.60 \pm 9.82$ & $16.32 \pm 17.29$ & 0.71 & 18 & $\mathrm{~ns}$ \\
Temperatura & $17.13 \pm 0.93$ & $19.03 \pm 0.57$ & -4.88 & 18 & $<0.001$ \\
\hline
\end{tabular}

ns: no significativo, $\mathrm{p}>0.05$

Los valores medios de clorofila- $a$ y biomasa de copépodos adultos de Neolovenula y Acanthocyclops mostraron valores más altos al final de nuestro estudio, sugiriendo la existencia de dos periodos diferentes (Fig. 3). Una prueba t de Student (Tabla 1) reflejó que la concentración de clorofila- $a$, la biomasa de estos copépodos adultos y la temperatura fueron significativamente más altas en el periodo comprendido entre los días 39-57 de este estudio (periodo 2) que en el periodo comprendido entre los días $0-36$ (periodo 1 ).
La fecundidad de Keratella y la biomasa de los individuos de Daphnia $>=1.2 \mathrm{~mm}$ no mostraron diferencias significativas entre ambos periodos (Tabla 1). Estas diferencias significativas de la concentración de clorofila- $a$, de la temperatura y de la biomasa de copépodos adultos entre los dos periodos considerados podrían determinar cambios en la dinámica poblacional de $K$. cochlearis en este sistema entre uno y otro periodo. Por esta razón, en este estudio analizamos en detalle la posible influencia de la disponibilidad de alimento

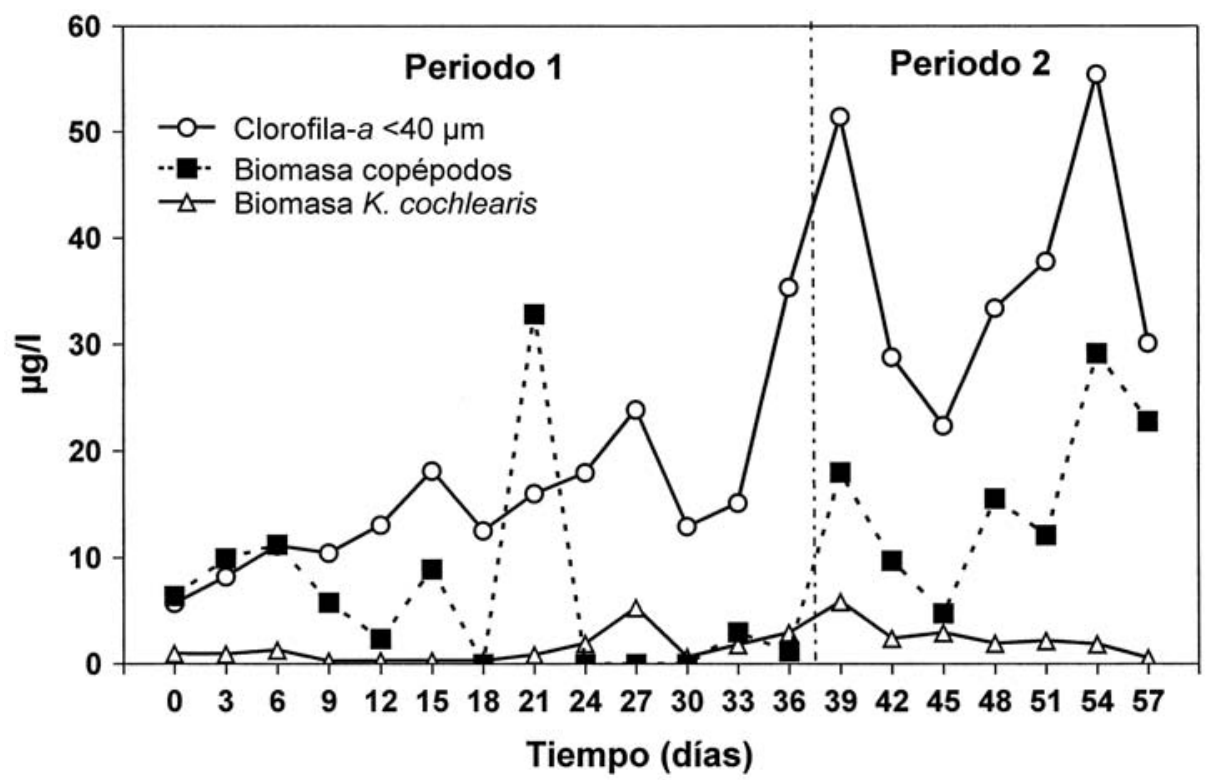

Figura 3. Variación temporal de los valores medios de clorofila- $a(<40 \mu \mathrm{m})$, biomasa de copépodos adultos (N. alluaudi y A. vernalis) y biomasa de K. cochlearis. Time-course of the mean values of chlorophyll-a concentration $(<40 \mu m)$, adult copepods biomass (N. alluaudi and A. vernalis) and $\mathrm{K}$. cochlearis biomass. 
PERIODO 1

PERIODO 2

a) Fecundidad de K. cochlearis ( $n^{\circ}$ huevos/ind)

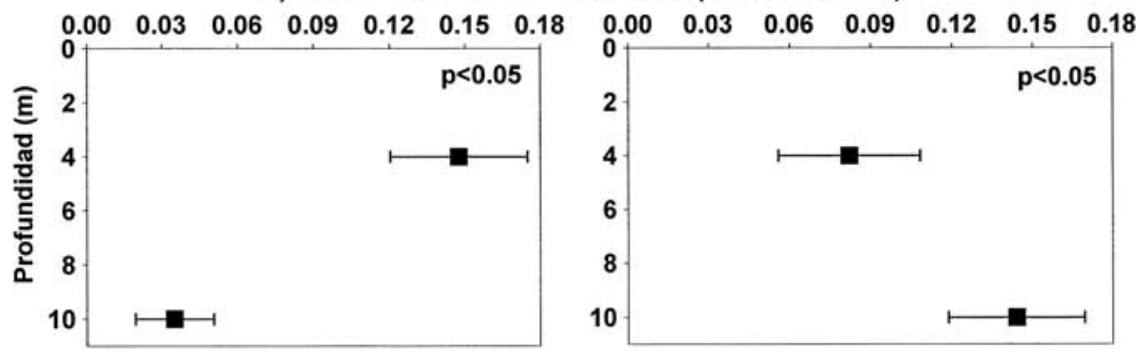

b) Clorofila-a $<40 \mu \mathrm{m}(\mu \mathrm{g} / \mathrm{l})$

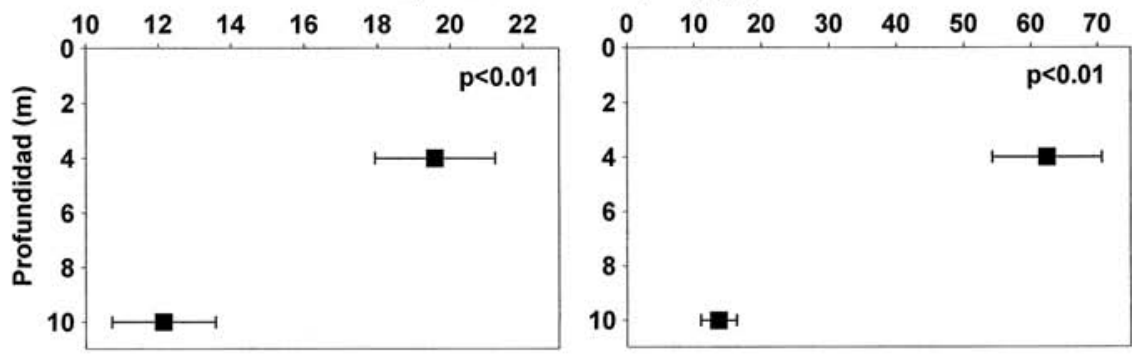

c) Biomasa de copépodos adultos $(\mu \mathrm{g} / \mathrm{l})$

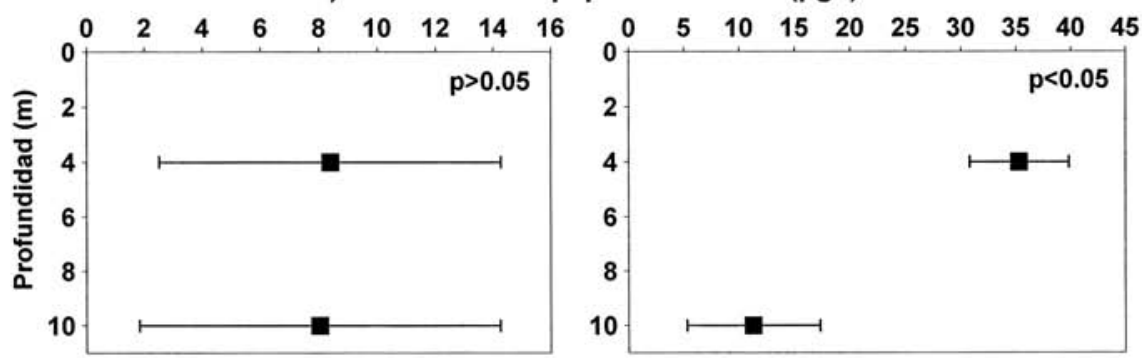

d) Ln (Densidad de $K$. cochlearis) (ind/l)
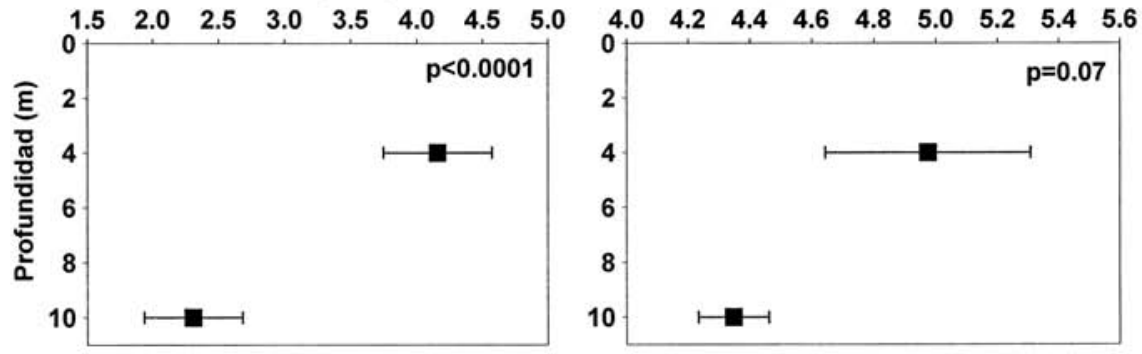

Figura 4. Valores medios ( $\pm \mathrm{EE}$ ) para cada periodo de la fecundidad de $K$. cochlearis (a), concentración de clorofila- $a<40 \mu \mathrm{m}$ (b), biomasa de copépodos adultos (c), y densidad de $K$. cochlearis (d), a $4 \mathrm{~m}$ y $10 \mathrm{~m}$ de profundidad. Las diferencias entre profundidades se analizaron mediante una prueba t de Student para muestras pareadas. Los análisis se restringieron a los valores de biomasa algal $(<40 \mu \mathrm{m})$ superiores a $0.5 \mu \mathrm{g} / \mathrm{ml}$ (periodo $1, \mathrm{n}=8$; periodo $2, \mathrm{n}=5$ ). Mean $( \pm S E)$ fecundity of $\mathrm{K}$. cochlearis (a) during each period, chlorophyll-a concentration $<40 \mu \mathrm{m}$ (b), adult copepods biomass (c), and density of $\mathrm{K}$. cochlearis (d), at $4 \mathrm{~m}$ and $10 \mathrm{~m}$ depth. Differences between depths were analysed using a t-test for paired observations. Analyses were restricted to times when algal biomass $(<40 \mu \mathrm{m}$ ) was higher than $0.5 \mu \mathrm{g} / \mathrm{ml}$ (period 1, $n=8$; period $2, n=5$ ). 
y la presencia de copépodos depredadores sobre la distribución espacio-temporal de la fecundidad de Keratella en cada uno de estos periodos.

Para aquellos momentos en que la biomasa algal $<40 \mu \mathrm{m}$ era superior a $0.5 \mu \mathrm{g} / \mathrm{ml}$, la fecundidad de $K$. cochlearis no mostró diferencias significativas entre superficie y $4 \mathrm{~m}$ de profundidad, ni durante el periodo $1(0 \mathrm{~m}: 0.129 \pm 0.031(\mathrm{EE})$; $4 \mathrm{~m}: 0.133 \pm 0.025(\mathrm{EE}) ; \mathrm{t}=-0.10$, g.l. $=11, \mathrm{p}=$ $0.09)$, ni durante en el periodo 2 (0 m: $0.099 \pm$ 0.026 (EE); $4 \mathrm{~m}: 0.113 \pm 0.028$ (EE); $\mathrm{t}=-0.77$, g.l. $=6, p=0.47)$. Sin embargo, la fecundidad de Keratella sí mostró diferencias significativas entre $4 \mathrm{~m}$ y $10 \mathrm{~m}$, tanto en el periodo 1 como en el periodo 2, y además con un patrón de distribución diferente entre ambos periodos (Fig. 4a).

Con el objetivo de analizar la posible influencia de la depredación por copépodos y de la competencia con grandes dáphnidos sobre la distribución vertical de la fecundidad de $K$. cochlearis en este sistema, en aquellos momentos en que la biomasa algal no era limitante para el crecimiento del rotífero, se realizaron análisis pareados entre profundidades. Ya que el número de casos comparables entre superficie y $4 \mathrm{~m}$ y, entre $4 \mathrm{~m}$ y $10 \mathrm{~m}$ no coincidieron, para simplificar los análisis estadísticos y su interpretación, en este trabajo nos centraremos en intentar explicar las diferencias de fecundidad existentes entre $4 \mathrm{~m} \mathrm{y}$ $10 \mathrm{~m}$ de profundidad en ambos periodos.

En el periodo 1, la fecundidad de Keratella fue significativamente más alta a $4 \mathrm{~m}$ que a 10 m (Fig. 4a), donde a su vez era más alta la concentración de clorofila- $a$ (Fig. 4b) y la temperatura $(\mathrm{p}<0.05)$. Ni la biomasa de copépodos adultos (Fig. 4c) ni la biomasa de los individuos de Daphnia con longitud $>=1.2 \mathrm{~mm}$ mostraron diferencias significativas $(p>0.05)$ entre $4 \mathrm{~m}$ y $10 \mathrm{~m}$ en este periodo. De hecho la fecundidad de Keratella estuvo correlacionada con la concentración de clorofila- $a$ en el periodo 1 $(\mathrm{n}=26 ; \mathrm{r}=0.41 ; \mathrm{p}<0.05)$, pero no así en el periodo 2 ( $\mathrm{p}>0.05)$. La fecundidad de Keratella no estuvo correlacionada con la temperatura en ningún periodo $(\mathrm{p}>0.05)$.

En el periodo 2, la fecundidad de Keratella invirtió su máximo de profundidad, siendo sig- nificativamente más baja a $4 \mathrm{~m}$ que a $10 \mathrm{~m}$ (Fig. 4a). Sin embargo, la concentración de clorofila- $a($ Fig. $4 \mathrm{~b})$ y la temperatura $(\mathrm{p}<0.01)$ seguían siendo significativamente más altas a $4 \mathrm{~m}$ que a $10 \mathrm{~m}$. Por el contrario, la biomasa de copépodos adultos fue, en este caso, significativamente más alta a $4 \mathrm{~m}$ que a $10 \mathrm{~m}$ (Fig. $4 \mathrm{c}$ ). La biomasa de los individuos de Daphnia con longitud $>=1.2 \mathrm{~mm}$ resultó ser significativamente más alta a $10 \mathrm{~m}$ en este periodo $(\mathrm{p}<0.05)$. La distribución vertical de la población de Keratella no cambió de forma apreciable entre ambos periodos (Fig. 4d).

\section{DISCUSIÓN}

La correlación observada entre la fecundidad de Keratella y la biomasa algal (Fig. 2) sugiere que, en este sistema, la importancia relativa del alimento sobre la fecundidad de Keratella podría verse reducida en los momentos en que la biomasa de los estadíos adultos de los copépodos depredadores, Neolovenula alluaudi y Acanthocyclops vernalis, alcanza valores más altos, incluso cuando la biomasa algal no está en concentraciones limitantes para el crecimiento de Keratella. Cuando la biomasa de los copépodos era relativamente baja, la disponibilidad de alimento y la temperatura podrían ser responsables de la distribución vertical observada de la fecundidad de K. cochlearis. En cambio, cuando la biomasa de estos copépodos depredadores alcanzó valores relativamente más altos, la importancia del alimento sobre la distribución vertical de la fecundidad de esta especie de rotífero podría verse reducida.

Las interacciones entre poblaciones están fuertemente influenciadas por la distribución espacial de las especies, de forma que las interacciones de competencia y depredación de crustáceos sobre rotíferos pueden intensificarse como consecuencia de un solapamiento espacial en la columna de agua entre los distintos taxones (véase p. ej. Williamson, 1993; González, 1998). En este sentido, el solapamiento espacial observado entre la población de Keratella y los 
copépodos adultos en el periodo 2 podría haber intensificado el efecto de la depredación selectiva sobre hembras reproductivas de $K$. cochlearis, o bien sobre los huevos de estas hembras, y por tanto, la importancia de la concentración de alimento y la temperatura sobre el patrón de distribución vertical de la fecundidad de esta especie de rotífero podría haber disminuido. Observaciones directas del comportamiento de alimentación de individuos adultos de estas dos especies de copépodos consideradas en este estudio de campo sobre hembras ovígeras de $K$. cochlearis, han puesto de manifiesto que, tanto Acanthocyclops vernalis como Neolovenula alluaudi pueden depredar sólo los huevos de Keratella, de forma que las hembras sobreviven, y también las hembras ovígeras completas (es decir, la hembra y el huevo) de Keratella (Ramos-Rodríguez \& Conde-Porcuna, datos no publicados). En consecuencia, ambas especies de depredadores invertebrados podrían reducir la fecundidad de $K$. cochlearis en este sistema. Algunos estudios experimentales han llegado a observar que otras especies de copépodos (Diacyclops thomasi y Diaptomus pallidus) depredan selectivamente los huevos de Keratella cochlearis sin consumir a las hembras que portan esos huevos (Williamson \& Butler, 1986; LeBlanc et al., 1997). Conde-Porcuna \& Sarma (1995) también mostraron, en un estudio de laboratorio, que el rotífero carnívoro Asplanchna girodi se alimentaba selectivamente sobre hembras reproductivas de $K$. cochlearis, y sugirieron que Asplanchna podría reducir la densidad de Keratella debido a una mayor mortalidad y a un descenso de las tasas de nacimiento de este rotífero. En situaciones naturales, también se ha observado que Asplanchna brightwelli depredaba selectivamente sobre hembras reproductivas de $K$. cochlearis en un sistema eutrófico, reduciendo la fecundidad del rotífero (Conde-Porcuna \& Declerck, 1998).

Por otro lado, la migración vertical diaria puede representar un mecanismo efectivo que minimice el riesgo de depredación por depredadores visuales y táctiles (véase p. ej. Lampert, 1989). Estos movimientos verticales pueden variar entre especies de rotíferos (Stewart \& George, 1987; González, 1998; Armengol \& Miracle, 2000). Algunos estudios han mostrado que $K$. cochlearis carece de este comportamiento de migración vertical en lagos profundos ( $\mathrm{p}$. ej. Ruttner-Kolisko, 1975; Pivoda, 1977; González, 1998). Sin embargo, otros estudios han señalado que esta especie de rotífero puede migrar verticalmente en lagos someros (CruzPizarro, 1978; Williamson \& Magnien, 1982). Stewart \& George (1987) mostraron que la amplitud de migración para $K$. cochlearis era muy baja (0.48-1.49 m). Galkovskaya \& Mityanina (1989) también han señalado que los movimientos migratorios de $K$. cochlearis son muy pequeños. De acuerdo con esto, en este estudio, la migración de esta especie de rotífero entre $4 \mathrm{~m}$ y $10 \mathrm{~m}$ de profundidad podría considerarse despreciable.

En resumen, este estudio muestra, en un embalse, como la distribución vertical de los valores de fecundidad de Keratella podría estar regulada no sólo por las condiciones de alimento, sino que también podría estar controlada por depredadores invertebrados. La competencia y/o interferencia por grandes cladóceros parece no tener importancia sobre la distribución vertical de la fecundidad de Keratella en este embalse. Además, observamos que la depredación por copépodos parece reducir la fecundidad de Keratella pero no la densidad de dicho rotífero, al menos de forma aparente. Esto sugiere que los copépodos tenderían a consumir los huevos de Keratella y no a las propias hembras que transportan estos huevos. La depredación selectiva e intensa de copépodos sobre los huevos de rotíferos es un factor importante que debería ser considerado en los estudios sobre dinámica espaciotemporal de las poblaciones de rotíferos.

\section{AGRADECIMIENTOS}

Este estudio estuvo financiado por una beca FPI del MEC (E. Ramos-Rodríguez) y por un proyecto de investigación de la Unión Europea (LIFE93/UK/3167). Agradecemos a A.M. Caro, 
J.M. Ramos y R. Ramos su colaboración en la toma de muestras.

\section{BIBLIOGRAFÍA}

APHA. 1992. Standard methods for the examination of water and wastewater, $18^{\mathrm{a}}$ ed. New York: APHA. ARMENGOL, X., A. ESPARCIA \& M. R. MIRACLE. 1998. Rotifer vertical distribution in a strongly stratified lake: a multivariate analysis. Hydrobiologia, 387/388: 161-170.

ARMENGOL, X. \& M. R. MIRACLE. 2000. Diel vertical movements of zooplankton in lake La Cruz (Cuenca, España). J. Plankton Res., 22: 1683-1703.

BOTTRELL, H. H., A. DUNCAN, Z. M. GLIWICZ, E. GRYGIEREK, A. HERZIG, A. HILLBRICHTILKOWSKA, H. KURASAWA, P. LARSSON \& T. WEGLENSKA. 1976. A review of some problems in zooplankton production studies. Norw. J. Zool., 24: 419-456.

CONDE-PORCUNA, J. M. \& S. DECLERCK. 1998. Regulation of rotifer species by invertebrate predators in a hypertrophic lake: selective predation on egg-bearing females and induction of morphological defences. J. Plankton Res., 20: 605-618.

CONDE-PORCUNA, J. M., R. MORALESBAQUERO \& L. CRUZ-PIZARRO. 1994. Effects of Daphnia longispina on rotifer populations in a natural environment: relative importance of food limitation and interference competition. $J$. Plankton Res., 16: 691-706.

CONDE-PORCUNA, J. M. \& S. S. S. SARMA. 1995. Prey selection by Asplanchna girodi (Rotifera): the importance of prey defence mechanisms. Freshwater Biol., 33: 341-348.

CRUZ-PIZARRO, L. 1978. Comparative vertical zonation and diurnal migration among Crustacea and Rotifera in the small high mountain lake La Caldera (Granada, Spain). Verh. Int. Ver. Limnol., 20: 1026-1032.

DIEGUEZ, M. C. \& J. J. GILBERT. 2002. Suppression of the rotifer Polyarthra remata by the omnivorous copepod Tropocyclops extensus: predation or competition. J. Plankton Res. 24: 359-369.

DUSSART, B. 1967. Les copépodes des eaux continentales d'Europe occidentale, Tome I, II. Paris: Boubée \& Cie.
EDMONDSON, W. T. 1965. Reproductive rates of planktonic rotifers as related to food and temperature in nature. Ecol. Monogr., 35: 61-111.

GALKOVSKAYA, G. A. \& I. F. MITYANINA. 1989. Morphological structure and functional patterns of Keratella cochlearis (Gosse) populations in stratified lakes. Hydrobiologia, 186/187: 119-128.

GILBERT, J. 1988. Suppression of rotifer populations by Daphnia: A review of the evidence, the mechanisms, and the effects on zooplankton community structure. Limnol. Oceanogr., 33: 1286-1303.

GONZÁLEZ, M. J. 1998. Spatial segregation between rotifers and cladocerans mediated by Chaoborus. Hydrobiologia, 387/388: 427-436.

HUNTER, M. D. \& P. W. PRICE. 1992. Playing chutes and ladders: heterogeneity and the relative roles of bottom-up and top-down forces in natural communities. Ecology, 73: 724-732.

LAMPERT, W. 1989. The adaptive significance of diel vertical migration of zooplankton. Funct. Ecol., 3: 21-27.

LEBLANC, J. S., W. D. TAYLOR \& O. E. JOHANNSSON. 1997. The feeding ecology of the cyclopoid copepod Diacyclops thomasi in Lake Ontario. J. Great Lakes Res., 23: 369-381.

OCDE (VOLLENWEIDER, R. A. \& J. J. KEREKES). 1982. Eutrophisation des eaux. Méthodes de surveillance, d'evaluation et de lutte. Paris: OCDE.

OOMS-WILMS, A. L., G. POSTEMA \& R. D. GULATI. 1999. Population dynamics of planktonic rotifers in Lake Loosdrecht, the Netherlands, in relation to their potential food and predators. Freshwater Biol., 42: 77-97.

PAUL, A. J. \& D. W. SCHINDLER. 1994. Regulation of rotifers by predatory calanoid copepods (subgenus Hesperodiatomus) in lakes of the Canadian Rocky Mountains. Can. J. Fish. Aquat. Sci., 51: 2520-2528.

PIVODA, B. 1977. Migration of planktonic rotifers in Lunzer Obersee (Austria). Arch. Hydrobiol. Beih. Ergebn. Limnol, 8: 50-52.

PLASSMANN, T., G. MAIER \& H. B. STICH. 1997. Predation impact of Cyclops vicinus on the rotifer community in Lake Constance in spring. $J$. Plankton Res., 19: 1069-1079.

POURRIOT, R \& C. ROUGIER. 1991. Importance volumétrique des oeufs chez les Rotifères planctoniques. Annls. Limnol., 27: 15-24.

ROCHE, K. 1990. Prey features affecting ingestion rates by Acanthocyclops robustus (Copepoda: Cyclopoida) on zooplankton. Oecologia, 83: 76-82. 
RUTTNER-KOLISKO, A. 1975. The vertical distribution of plankton rotifers in a small alpine lake with a sharp oxygen depletion (Lunzer Obersee). Verh. Int. Ver. Limnol., 19: 1286-1294.

SOKAL, R. R. \& F. J. ROHLF. 1995. Biometry, $3^{\mathrm{a}}$ ed. New York: W. H. Freeman \& Company.

STEMBERGER, R. S. \& M. S. EVANS. 1984. Rotifer seasonal succession and copepod predation in Lake Michigan. J. Great Lakes Res., 10: 417-428

STEWART, L. J. \& D. G. GEORGE, 1987. Environmental factors influencing the vertical migration of plankton rotifers in a hypereutrophic tarn. Hydrobiologia, 147: 203-208.

TALLING, J. F. \& D. DRIVER. 1961. Some problems in the estimation of chlorophyll- $a$ in phytoplankton. Proceedings of a Conference on Primary Productivity Measurements, Marine and Freshwater. US Atomic Energy Comm. Public. TID-7633, pp. 142-146.
WILLIAMSON, C. E. 1993. Linking predation risk models with behavioural mechanisms: identifying population bottlenecks. Ecology, 74: 320-331.

WILLIAMSON, C. E. \& N. M. BUTLER. 1986. Predation on rotifers by the suspension-feeding calanoid copepod Diaptomus pallidus. Limnol. Oceanogr., 31: 393-402.

WILLIAMSON, C. E. \& R. E. MAGNIEN. 1982. Diel vertical migration in a Mesocyclops edax: Implications for predation rate estimates. $J$. Plankton Res., 4: 329-339.

YAN, N. D., W. KELLER, H. J. MACISAAC \& L. J. MCEACHERN. 1991. Regulation of zooplankton community structure of an acidified lake by Chaoborus. Ecol. Appl., 1: 52-65.

ZIMMERMAN, V. C. 1974. Die pelagischen Rotatorien des Sempachersees, mit spezieller Berucksichtigung der Brachionden und der Ernährungsfrage. Schweiz. Z. Hydrol., 36: 205300. 